\title{
TRIPOLAR FUZZY SOFT IDEALS AND TRIPOLAR FUZZY SOFT INTERIOR IDEALS OVER $\Gamma$-SEMIRING
}

\author{
M. Murali Krishna Rao and B. Venkateswarlu
}

(C) 2020 by University of Niš, Serbia | Creative Commons License: CC BY-NC-ND

\begin{abstract}
In this paper, we have introduced the notion of tripolar fuzzy soft $\Gamma$-subsemiring, tripolar fuzzy soft ideal, tripolar fuzzy soft interior ideals over $\Gamma$-semiring and also studied some of their algebraic properties and the relations between them.

Keywords: tripolar fuzzy set, soft set, fuzzy soft set, tripolar fuzzy soft ideal, tripolar fuzzy soft interior ideal.
\end{abstract}

\section{Introduction}

In 1995, Murali Krishna Rao $[16,17]$ introduced the notion of a $\Gamma$-semiring as a generalization of $\Gamma$-ring, ring, ternary semiring and semiring. As a generalization of ring, the notion of a $\Gamma$-ring was introduced by Nobusawa [22] in 1964. As a generalization of ring, semiring was introduced by Vandiver [25] in 1934. In 1981, Sen [24] introduced the notion of $\Gamma$-semigroup as a generalization of semigroup. The notion of ternary algebraic system was introduced by Lehmer [12] in 1932. Lister [13] introduced ternary ring. The set of all negative integers $Z$ is not a semiring with respect to usual addition and multiplication but $Z$ forms a $\Gamma$-semiring where $\Gamma=Z$. The important reason for the development of $\Gamma$-semiring is a generalization of results of rings, $\Gamma$-rings, semirings, semigroups and ternary semirings. Murali Krishna Rao and Venkateswarlu [21] introduced the notion of $\Gamma$-incline and field $\Gamma$-semiring and studied properties of regular $\Gamma$-incline and field $\Gamma$-semiring.

The theory of fuzzy sets is the most appropriate theory for dealing with uncertainty was first introduced by Zadeh [26]. Rosenfeld [23] introduced fuzzy group. There are many extensions of fuzzy sets, for example, intuitionistic fuzzy sets, interval valued fuzzy sets, vague sets, bipolar fuzzy sets, cubic sets etc. Molodtsov [15] introduced the concept of soft set theory as a new mathematical tool for dealing with uncertainties. Feng et al. [5] initiated the study of soft semirings. Soft rings are defined by Acar et al. [1] and Ghosh et al. [6] who initiated the study of fuzzy soft rings and fuzzy soft ideals. In 2001, Maji et al. [14] combined the concept of

Received October 25, 2017; accepted March 6, 2020

2010 Mathematics Subject Classification. Primary 08A72; Secondary 16 Y60. 
fuzzy set theory which was introduced by Zadeh [26] in 1965 and the notion of soft set theory which was introduced by Molodstov [15] in 1999. Aktas and Cagman [2] introduced the concept of fuzzy subgroup, soft sets and soft groups which was extended by Aygnnogln and Aygun [4]. Murali Krishna Rao [18] studied fuzzy soft $\Gamma$-semiring homomorphism, fuzzy soft $\Gamma$-semiring and fuzzy soft $k$-ideal over $\Gamma$-semiring, In 2000, Lee $[10,11]$ used the term bipolar valued fuzzy sets and applied it to algebraic structure. Bipolar fuzzy sets are an extension of fuzzy sets whose membership degree range is $[-1,1]$. In 1994, Zhang [27] initiated the concept bipolar fuzzy sets as a generalization of fuzzy sets. Jun et al. [7, 8] introduced the notion of bipolar fuzzy ideals and bipolar fuzzy filters in CI-algebras. The ideals of " an intuitionistic fuzzy set" was first introduced by Atanassor [3] as a generalization of notion of fuzzy set. Murali Krishna Rao [19] introduced the notion of tripolar fuzzy set to be able to deal with tripolar information as a generalization of fuzzy set, bipolar fuzzy set and intuitionistic fuzzy set and introduced the notion of tripolar fuzzy ideals and tripolar fuzzy interior ideals of $\Gamma$-semigoup. In this paper, we have introduced the notion of tripolar fuzzy soft ideals and interior ideals over $\Gamma$-semiring. We have studied some of their algebraic properties and the relations between them.

\section{Preliminaries}

In this section, we recall some definitions introduced by the pioneers in this field earlier.

Definition 2.1. Let $(M,+)$ and $(\Gamma,+)$ be commutative semigroups. Then we call $M$ as a $\Gamma$-semiring,if there exists a mapping $M \times \Gamma \times M \rightarrow M$ is written $(x, \alpha, y)$ as $x \alpha y$ such that it satisfies the following axioms:

(i) $x \alpha(y+z)=x \alpha y+x \alpha z$

(ii) $(x+y) \alpha z=x \alpha z+y \alpha z$

(iii) $x(\alpha+\beta) y=x \alpha y+x \beta y$

(iv) $x \alpha(y \beta z)=(x \alpha y) \beta z$, for all $x, y, z \in M$ and $\alpha, \beta \in \Gamma$.

Every semiring $R$ is a $\Gamma$-semiring with $\Gamma=R$ and ternary operation $x \gamma y$ as the usual semiring multiplication.

Example 2.1. Let $M$ be a a set of all rational numbers and $\Gamma$ be a set of all natural numbers are commutative semigroups with respect to usual addition. Define the mapping $M \times \Gamma \times M \rightarrow M$ by $a \alpha b$ as usual multiplication, forall $a, b \in M, \alpha \in \Gamma$. Then $M$ is a $\Gamma$-semiring.

Definition 2.2. A $\Gamma$-semiring $M$ is said to have zero element if there exists an element $0 \in M$ such that $0+x=x=x+0$ and $0 \alpha x=x \alpha 0=0$, forall $x \in M, \alpha \in \Gamma$. 
Definition 2.3. Let $M$ be a $\Gamma$-semiring. An element $1 \in M$ is said to be unity if for each $x \in M$ there exists $\alpha \in \Gamma$ such that $x \alpha 1=1 \alpha x=x$.

Definition 2.4. A $\Gamma$-semiring $M$ is said to be commutative $\Gamma$-semiring if $x \alpha y=$ $y \alpha x$, for all $x, y \in M$ and $\alpha \in \Gamma$.

Definition 2.5. Let $M$ be a $\Gamma$-semiring. An element $a \in M$ is said to be regular element of $M$ if there exist $x \in M, \alpha, \beta \in \Gamma$ such that $a=a \alpha x \beta a$.

Definition 2.6. Let $M$ be a $\Gamma$-semiring. If every element of $M$ is a regular then $M$ is said to be regular $\Gamma$-semiring.

Definition 2.7. A non-empty subset $A$ of $\Gamma$-semiring $M$ is called

(i) a $\Gamma$-subsemiring of $M$ if $(A,+)$ is a subsemigroup of $(M,+)$ and $A \Gamma A \subseteq A$.

(ii) a quasi ideal of $M$ if $A$ is a $\Gamma$-subsemiring of $M$ and $A \Gamma M \cap M \Gamma A \subseteq A$.

(iii) a bi-ideal of $M$ if $A$ is a $\Gamma$-subsemiring of $M$ and $A \Gamma M \Gamma A \subseteq A$.

(iv) an interior ideal of $M$ if $A$ is a $\Gamma$-subsemiring of $M$ and $M \Gamma A \Gamma M \subseteq A$.

(v) a left (right) ideal of $M$ if $A$ is a $\Gamma$-subsemiring of $M$ and $M \Gamma A \subseteq A(A \Gamma M \subseteq$ A).

(vi) an ideal if $A$ is a $\Gamma$-subsemiring of $M, A \Gamma M \subseteq A$ and $M \Gamma A \subseteq A$.

(vii) a $k$-ideal if $A$ is a $\Gamma$-subsemiring of $M, A \Gamma M \subseteq A, M \Gamma A \subseteq A$ and $x \in$ $M, x+y \in A, y \in A$ then $x \in A$.

Definition 2.8. A tripolar fuzzy set $A$ in a universe set $X$ is an object having the form $A=\left\{\left(x, \mu_{A}(x), \lambda_{A}(x), \delta_{A}(x)\right) \mid x \in X\right.$ and $\left.0 \leq \mu_{A}(x)+\lambda_{A}(x) \leq 1\right\}$, where $\mu_{A}: X \rightarrow[0,1] ; \lambda_{A}: X \rightarrow[0,1] ; \delta_{A}: X \rightarrow[-1,0]$ such that $0 \leq \mu_{A}(x)+\lambda_{A}(x) \leq 1$. The membership degree $\mu_{A}(x)$ characterizes the extent that the element $x$ satisfies to the property corresponding to tripolar fuzzy set $\mathrm{A}, \lambda_{A}(x)$ characterizes the extent that the element $x$ satisfies to the not property(irrelevant ) corresponding to tripolar fuzzy set $\mathrm{A}$ and $\delta_{A}(x)$ characterizes the extent that the element $x$ satisfies to the implicit counter property of tripolar fuzzy set A. For simplicity $A=\left(\mu_{A}, \lambda_{A}, \delta_{A}\right)$ has been used for $A=\left\{\left(x, \mu_{A}(x), \lambda_{A}(x), \delta_{A}(x)\right) \mid x \in X, 0 \leq \mu_{A}(x)+\lambda_{A}(x) \leq 1\right\}$.

Remark 2.1. A tripolar fuzzy set $A$ is a generalization of a bipolar fuzzy set and an intuitionistic fuzzy set. A tripolar fuzzy set $A=\left\{\left(x, \mu_{A}(x), \lambda_{A}(x), \delta_{A}(x) \mid x \in X\right)\right.$ represents the sweet taste of food stuffs. Assuming the sweet taste of food stuff as a positive membership value $\mu_{A}(x)$, i.e. the element $x$ is satisfying the sweet property. Then bitter taste of food stuff as a negative membership value $\delta_{A}(x$,$) i.e. the element x$ is satisfying the bitter property, and the remaining tastes of food stuffs like acidic, chilly etc., as a non memberships value $\lambda_{A}(x)$, i.e., the element is satisfying irrelevant to the sweet property. 
Definition 2.9. A tripolar fuzzy set $A=\left(\mu_{A}, \lambda_{A}, \delta_{A}\right)$ of a $\Gamma$-semiring $M$ is called a tripolar fuzzy $\Gamma$-subsemiring of $M$ if $A$ satisfies the following conditions.

(i) $\mu_{A}(x+y) \geq \min \left\{\mu_{A}(x), \mu_{A}(y)\right\}$

(ii) $\lambda_{A}(x+y) \leq \max \left\{\lambda_{A}(x), \lambda_{A}(y)\right\}$

(iii) $\delta_{A}(x+y) \leq \max \left\{\delta_{A}(x), \delta_{A}(y)\right\}$

(iv) $\mu_{A}(x \alpha y) \geq \min \left\{\mu_{A}(x), \mu_{A}(y)\right\}$

(v) $\lambda_{A}(x \alpha y) \leq \max \left\{\lambda_{A}(x), \lambda_{A}(y)\right\}$

(vi) $\delta_{A}(x \alpha y) \leq \max \left\{\delta_{A}(x), \delta_{A}(y)\right\}$, for all $x, y, z \in M, \alpha \in \Gamma$.

Definition 2.10. A tripolar fuzzy $\Gamma$-subsemiring $A=\left(\mu_{A}, \lambda_{A}, \delta_{A}\right)$ of a $\Gamma$-semiring $M$ is called a tripolar fuzzy ideal of $M$ if $A$ satisfies the following conditions.

(i) $\mu_{A}(x \alpha y) \geq \max \left\{\mu_{A}(x), \mu_{A}\right)(x y\}$

(ii) $\lambda_{A}(x \alpha y) \leq \min \left\{\lambda_{A}(x), \lambda_{A}(y)\right\}$

(iii) $\delta_{A}(x \alpha y) \leq \min \left\{\delta_{A}(x), \delta_{A}(y)\right\}$, for all $x, y, z \in M, \alpha \in \Gamma$

Definition 2.11. A tripolar fuzzy $\Gamma$-subsemiring $A=\left(\mu_{A}, \lambda_{A}, \delta_{A}\right)$ of a $\Gamma$-semiring $M$ is called a tripolar fuzzy interior ideal of $M$ if $A$ satisfies the following conditions.

(i) $\mu_{A}(x \alpha z \beta y) \geq \mu_{A}(z)$

(ii) $\lambda_{A}(x \alpha z \beta y) \leq \lambda_{A}(z)$

(iii) $\delta_{A}(x \alpha z \beta y) \leq \delta_{A}(z)$, for all $x, y, z \in M, \alpha, \beta \in \Gamma$.

Definition 2.12. Let $(f, A),(g, B)$ be fuzzy soft sets over a $\Gamma$-semiring $M$. The intersection of fuzzy soft sets $(f, A)$ and $(g, B)$ is denoted by $(f, A) \cap(g, B)=(h, C)$ where $C=A \cup B$ is defined as

$$
h_{c}= \begin{cases}f_{c}, & \text { if } c \in A \backslash B ; \\ g_{c}, & \text { if } c \in B \backslash A ; \\ f_{c} \cap g_{c}, & \text { if } c \in A \cap B .\end{cases}
$$

for all $c \in A \cup B$ and $x \in M$.

Definition 2.13. Let $(f, A),(g, B)$ be fuzzy soft sets over a $\Gamma$-semiring $M S$. The union of fuzzy soft sets $(f, A)$ and $(g, B)$ is denoted by $(f, A) \cup(g, B)=(h, C)$ where $C=A \cup B$ is defined as

$$
h_{c}=\left\{\begin{array}{ll}
f_{c}, & \text { if } c \in A \backslash B ; \\
g_{c}, & \text { if } c \in B \backslash A ; \\
f_{c} \cup g_{c}, & \text { if } c \in A \cap B .
\end{array},\right.
$$

for all $c \in A \cup B$ and $x \in M$. 
Definition 2.14. Let $(f, A),(g, B)$ be fuzzy soft sets over a $\Gamma$-semiring $M$.

" $(f, A)$ and $(g, B)$ is denoted by " $(f, A) \wedge(g, B)$ " is defined by $(f, A) \wedge(g, B)=(h, C)$, where $C=A \times B$. $h_{c}(x)=\min \left\{f_{a}(x), g_{b}(x)\right\}$ for all $c=(a, b) \in A \times B$ and $x \in M$.

Definition 2.15. Let $(f, A),(g, B)$ be fuzzy soft sets over an ordered $\Gamma$-semiring $M$. " $(f, A) \operatorname{or}(g, B)$ " is denoted by $(f, A) \vee(g, B)$ is defined by $(f, A) \vee(g, B)=(h, C)$, where $C=A \times B$ and $h_{c}(x)=\max \left\{f_{a}(x), g_{b}(x)\right\}$, for all $c=(a, b) \in A \times B, x \in U$.

\section{TRIPOLAR FUZZY SOFT IDEALS OVER $\Gamma$-SEMIRING}

In this section, we introduce the notion of tripolar fuzzy sets to be able to deal with tripolar information as a generalization of fuzzy sets, bipolar fuzzy set and intuitionistic fuzzy sets. We introduce the notion of tripolar fuzzy soft ideals and interior ideals over $\Gamma$-semiring.

Definition 3.1. A tripolar fuzzy soft set $(f, A)$ over $\Gamma$-semiring $M$ is called a tripolar fuzzy soft $\Gamma$-semiring over $M$ if $f(a)=\left\{\mu_{f(a)}(x), \lambda_{f(a)}(x), \delta_{f(a)}(x) \mid x \in\right.$ $M, a \in A\}$, where $\mu_{f(a)}(x): M \rightarrow[0,1] ; \lambda_{f(a)}(x): M \rightarrow[0,1] ; \delta_{f(a)}(x): M \rightarrow$ $[-1,0]$ such that $0 \leq \mu_{f(a)}(x)+\lambda_{f(a)}(x) \leq 1$ and for all $x \in M$ satisfying the following conditions

(i) $\mu_{f(a)}(x+y) \geq \min \left\{\mu_{f(a)}(x), \mu_{f(a)}(y)\right\}$

(ii) $\lambda_{f(a)}(x+y) \leq \max \left\{\lambda_{f(a)}(x), \lambda_{f(a)}(y)\right\}$

(iii) $\delta_{f(a)}(x+y) \leq \max \left\{\delta_{f(a)}(x), \delta_{f(a)}(y)\right\}$

(iv) $\mu_{f(a)}(x \alpha y) \geq \min \left\{\mu_{f(a)}(x), \mu_{f(a)}(y)\right\}$

(v) $\lambda_{f(a)}(x \alpha y) \leq \max \left\{\lambda_{f(a)}(x), \lambda_{f(a)}(y)\right\}$

(vi) $\delta_{f(a)}(x \alpha y) \leq \max \left\{\delta_{f(a)}(x), \delta_{f(a)}(y)\right\}$, for all $x, y \in M, \alpha \in \Gamma$ and $a \in A$.

Definition 3.2. A tripolar fuzzy soft set $(f, A)$ over a $\Gamma$-semiring $M$ is called a tripolar fuzzy soft ideal over $M$ if

(i) $\mu_{f(a)}(x+y) \geq \min \left\{\mu_{f(a)}(x), \mu_{f(a)}(y)\right\}$

(ii) $\lambda_{f(a)}(x+y) \leq \max \left\{\lambda_{f(a)}(x), \lambda_{f(a)}(y)\right\}$

(iii) $\delta_{f(a)}(x+y) \leq \max \left\{\delta_{f(a)}(x), \delta_{f(a)}(y)\right\}$

(iv) $\mu_{f(a)}(x \alpha y) \geq \max \left\{\mu_{f(a)}(x), \mu_{f(a)}(y)\right\}$

(v) $\lambda_{f(a)}(x \alpha y) \leq \min \left\{\lambda_{f(a)}(x), \lambda_{f(a)}(y)\right\}$

(vi) $\delta_{f(a)}(x \alpha y) \leq \min \left\{\delta_{f(a)}(x), \delta_{f(a)}(y)\right\}$, for all $x, y \in M, \alpha \in \Gamma$ and $a \in A$. 
Remark 3.1. Every tripolar fuzzy soft ideal $(f, A)$ over a $\Gamma$-semiring $M$ is a tripolar fuzzy soft semiring $(f, A)$ over $M$ but the converse is not true.

Example 3.1. Let $M=\left\{x_{1}, x_{2}, x_{3}\right\}, \Gamma=\{\alpha, \beta\}$. Then, we shall define the operations with the following tables:

\begin{tabular}{|c|c|c|}
\hline+ & $\alpha$ & $\beta$ \\
\hline$\alpha$ & $\alpha$ & $\alpha$ \\
\hline$\beta$ & $\alpha$ & $\beta$ \\
\hline
\end{tabular}

\begin{tabular}{|c|l|l|l|}
\hline+ & $x_{1}$ & $x_{2}$ & $x_{3}$ \\
\hline$x_{1}$ & $x_{1}$ & $x_{2}$ & $x_{3}$ \\
\hline$x_{2}$ & $x_{2}$ & $x_{2}$ & $x_{3}$ \\
\hline$x_{3}$ & $x_{3}$ & $x_{3}$ & $x_{2}$ \\
\hline
\end{tabular}

\begin{tabular}{|c|l|l|l|}
\hline$\alpha$ & $x_{1}$ & $x_{2}$ & $x_{3}$ \\
\hline$x_{1}$ & $x_{1}$ & $x_{3}$ & $x_{3}$ \\
\hline$x_{2}$ & $x_{3}$ & $x_{2}$ & $x_{3}$ \\
\hline$x_{3}$ & $x_{3}$ & $x_{3}$ & $x_{3}$ \\
\hline
\end{tabular}

\begin{tabular}{|c|c|c|c|}
\hline$\beta$ & $x_{1}$ & $x_{2}$ & $x_{3}$ \\
\hline$x_{1}$ & $x_{1}$ & $x_{2}$ & $x_{3}$ \\
\hline$x_{2}$ & $x_{3}$ & $x_{2}$ & $x_{3}$ \\
\hline$x_{3}$ & $x_{3}$ & $x_{3}$ & $x_{3}$ \\
\hline
\end{tabular}

Let $E=\{a, b, c\}$ and $B=\{a, b\}$. Then $(\phi, B)$ is tripolar fuzzy soft set defined as $(\phi, B)=\{\phi(a), \phi(b)\}$, where

$\phi(a)=\left\{\left(x_{1}, 0.2,0.7,-0.2\right),\left(x_{2}, 0.3,0.6,-0.3\right),\left(x_{3}, 0.6,0.3,-0.3\right)\right\}$

$\phi(b)=\left\{\left(x_{1}, 0.4,0.5,-0.3\right),\left(x_{2}, 0.6,0.3,-0.5\right),\left(x_{3}, 0.5,0.4,-0.2\right)\right\}$.

Then $(\phi, B)$ is a tripolar fuzzy soft $\Gamma$-subsemiring over $M$ and $(\phi, B)$ is not a tripolar fuzzy soft ideal over $M$.

$(\phi, B)$ is tripolar fuzzy soft interior ideal over $M$.

Definition 3.3. A tripolar fuzzy soft $(f, A)$ over $\Gamma$-semiring $M$ is called a tripolar fuzzy soft interior ideal of $M$ if

(i) $\mu_{f(a)}(x \alpha z \beta y) \geq \mu_{f(a)}(z)$

(ii) $\quad \lambda_{f(a)}(x \alpha z \beta y) \leq \lambda_{f(a)}(z)$

(iii) $\quad \delta_{f(a)}(x \alpha z \beta y) \leq \delta_{f(a)}(z)$, for all $x, y, z \in M, \alpha, \beta \in \Gamma$ and $a \in A$.

Theorem 3.1. Every tripolar fuzzy soft ideal over a $\Gamma$-semiring Mis a tripolar fuzzy soft interior ideal over a $\Gamma$-semiring $M$.

Proof. Let $(f, A)$ be tripolar fuzzy soft ideal over a $\Gamma$-semiring $M$. Then $f(a)=$ $\{\mu(a), \lambda(a), f(a)\}$ is a tripolar fuzzy ideal of $M, a \in A$. Then

(i) $\mu_{f(a)}(x \alpha z \beta y) \geq \mu_{f(a)}(x \alpha z) \geq \mu_{f(a)}(z)$

(ii) $\lambda_{f(a)}(x \alpha z \beta y) \leq \lambda_{f(a)}(x \alpha z) \leq \lambda_{f(a)}(z)$

(iii) $\delta_{f(a)}(x \alpha z \beta y) \leq \delta_{f(a)}(x \alpha z) \leq \delta_{f(a)}(z)$, forall $x, y, z \in M, \alpha, \beta \in \Gamma$ and $a \in A$.

Hence $(f, A)$ is a tripolar fuzzy soft interior ideal over $M$.

Theorem 3.2. Every tripolar fuzzy soft interior ideal over a regular $\Gamma$-semiring $M$ is a tripolar fuzzy soft ideal over $M$.

Proof. Let $(f, A)$ be tripolar fuzzy soft interior ideal over a regular $\Gamma$-semiring $M$. Then $f(a)=\left\{\mu_{f(a)}, \lambda_{f(a)}, \delta_{f(a)}\right\}$ is a tripolar fuzzy ideal of $M, a \in A$. 
Suppose $x, y \in M, \alpha \in \Gamma$. Then $x \alpha y \in M$. Then there exist $\beta, \gamma \in \Gamma, z \in M$ such that $x \alpha y=x \alpha y \beta z \gamma x \alpha y$.

$$
\begin{aligned}
\mu_{f(a)}(x \alpha y) & =\mu_{f(a)}(x \alpha y \beta z \gamma x \alpha y) \\
& =\mu_{f(a)}(x \alpha y \beta(z \gamma x \alpha y)) \\
& \geq \mu_{f(a)}(y) \\
\mu_{f(a)}(x \alpha y) & =\mu_{f(a)}((x \alpha y \beta z) \gamma x \alpha y) \\
& \geq \mu_{f(a)}(x) .
\end{aligned}
$$

Hence $\mu_{f(a)}$ is a fuzzy ideal of $M$.

$$
\begin{aligned}
\lambda_{f(a)}(x \alpha y) & =\lambda_{f(a)}(x \alpha y \beta z \gamma x \alpha y) \\
& \leq \lambda_{f(a)}(y) \\
\lambda_{f(a)}(x \alpha y) & =\lambda_{f(a)}((x \alpha y \beta z) \gamma x \alpha y) \\
& \leq \lambda_{f(a)}(x)
\end{aligned}
$$

Hence $\lambda_{f(a)}$ is a fuzzy ideal of $M$.

$$
\begin{aligned}
\delta_{f(a)}(x \alpha y) & =\delta_{f(a)}(x \alpha y \beta z \gamma x \alpha y) \\
& \leq \delta_{f(a)}(y) \\
\delta_{f(a)}(x \alpha y) & \leq \delta_{f(a)}(x) .
\end{aligned}
$$

Hence $\delta_{f(a)}$ is a fuzzy ideal of $M$.

Therefore, $f(a)$ is a tripolar fuzzy ideal of $\Gamma$-semiring $M$.

Thus, $(f, A)$ is a tripolar fuzzy soft ideal of $\Gamma$-semiring $M$.

Theorem 3.3. If $(f, A)$ and $(g, B)$ are two tripolar fuzzy soft ideals over a $\Gamma$-semiring $M$ then $(f, A) \wedge(g, B)$ is a tripolar fuzzy soft ideal over a $\Gamma$-semiring $M$.

Proof. Suppose $(f, A)$ and $(g, B)$ are two tripolar fuzzy soft ideals over a $\Gamma$-semiring $M$.

Then by Definition [2.14], $(f, A) \wedge(g, B)=(f \cap g, C)$, where $C=A \times B$ and $(f \wedge g)(a, b)=f(a) \cap g(b)$, for all $(a, b) \in C$. Then

$$
\begin{aligned}
\mu_{f(a) \cap g(b)}(x+y) & =\min \left\{\mu_{f(a)}(x+y), \mu_{g(b)}(x+y)\right\} \\
& \geq \min \left\{\min \left\{\mu_{f(a)}(x), \mu_{f(a)}(y)\right\}, \min \left\{\mu_{g(b)}(x), \mu_{g(b)}(y)\right\}\right\} \\
& =\min \left\{\min \left\{\mu_{f(a)}(x), \mu_{g(b)}(x)\right\}, \min \left\{\mu_{f(a)}(y), \mu_{g(b)}(y)\right\}\right\} \\
& =\min \left\{\mu_{f(a) \cap g(b)}(x), \mu_{f(a) \cap g(b)}(y)\right\} . \\
\lambda_{(f \cap g)(a, b)}(x+y) & =\min \left\{\lambda_{f(a)}(x+y), \lambda_{g(b)}(x+y)\right\} \\
& \geq \min \left\{\max \left\{\lambda_{f(a)}(x), \lambda_{f(a)}(y)\right\}, \max \left\{\lambda_{g(b)}(x), \lambda_{g(b)}(y)\right\}\right\} \\
& =\max \left\{\min \left\{\lambda_{f(a)}(x), \lambda_{g(b)}(x)\right\}, \min \left\{\lambda_{f(a)}(y), \lambda_{g(b)}(y)\right\}\right\} \\
& =\max \left\{\lambda_{f(a) \cap g(b)}(x), \lambda_{f(a) \cap g(b)}(y)\right\} .
\end{aligned}
$$




$$
\begin{aligned}
\delta_{(f \cap g)(a, b)}(x+y) & =\min \left\{\delta_{f(a)}(x+y), \delta_{g(b)}(x+y)\right\} \\
& \geq \min \left\{\max \left\{\delta_{f(a)}(x), \delta_{f(a)}(y)\right\}, \max \left\{\delta_{g(b)}(x), \delta_{g(b)}(y)\right\}\right\} \\
& =\max \left\{\min \left\{\delta_{f(a)}(x), \delta_{g(b)}(x)\right\}, \min \left\{\delta_{f(a)}(y), \delta_{g(b)}(y)\right\}\right\} \\
& =\max \left\{\delta_{f(a) \cap g(b)}(x), \delta_{f(a) \cap g(b)}(y)\right\} . \\
\mu_{f(a) \cap g(b)}(x \alpha y) & =\min \left\{\mu_{f(a)}(x \alpha y), \mu_{g(b)}(x \alpha y)\right\} \\
& \geq \min \left\{\min \left\{\mu_{f(a)}(x), \mu_{f(a)}(y)\right\}, \min \left\{\mu_{g(b)}(x), \mu_{g(b)}(y)\right\}\right\} \\
& =\min \left\{\min \left\{\mu_{f(a)}(x), \mu_{g(b)}(x)\right\}, \min \left\{\mu_{f(a)}(y), \mu_{g(b)}(y)\right\}\right\} \\
& =\min \left\{\mu_{f(a) \cap g(b)}(x), \mu_{f(a) \cap g(b)}(y)\right\} . \\
& =\min \left\{\lambda_{f(a)}(x \alpha y), \lambda_{g(b)}(x \alpha y)\right\} \\
\lambda_{(f \cap g)(a, b)}(x \alpha y) & \min \left\{\max \left\{\lambda_{f(a)}(x), \lambda_{f(a)}(y)\right\}, \max \left\{\lambda_{g(b)}(x), \lambda_{g(b)}(y)\right\}\right\} \\
& =\max \left\{\min \left\{\lambda_{f(a)}(x), \lambda_{g(b)}(x)\right\}, \min \left\{\lambda_{f(a)}(y), \lambda_{g(b)}(y)\right\}\right\} \\
& =\max \left\{\lambda_{f(a) \cap g(b)}(x), \lambda_{f(a) \cap g(b)}(y)\right\} . \\
\delta_{(f \cap g)(a, b)}(x \alpha y) & =\min \left\{\delta_{f(a)}(x \alpha y), \delta_{g(b)}(x \alpha y)\right\} \\
\leq & \min \left\{\max \left\{\delta_{f(a)}(x), \delta_{f(a)}(y)\right\}, \max \left\{\delta_{g(b)}(x), \delta_{g(b)}(y)\right\}\right\} \\
& =\max \left\{\min \left\{\delta_{f(a)}(x), \delta_{g(b)}(x)\right\}, \min \left\{\delta_{f(a)}(y), \delta_{g(b)}(y)\right\}\right\} \\
& =\max \left\{\delta_{f(a) \cap g(b)}(x), \delta_{f(a) \cap g(b)}(y)\right\} .
\end{aligned}
$$

Hence $(f, A) \wedge(g, B)$ is a tripolar fuzzy soft ideal over a $\Gamma$-semiring $M$.

Theorem 3.4. If $(f, A)$ and $(g, B)$ are two tripolar fuzzy soft interior ideals over $a \Gamma$-semiring $M$ then $(f, A) \wedge(g, B)$ is a tripolar fuzzy interior ideals of $\Gamma$-semiring $M$.

Proof. Suppose $(f, A)$ and $(g, B)$ are two tripolar fuzzy soft interior ideals over a $\Gamma$-semiring $M$.

Then by Theorem[3.3], $(f, A) \wedge(g, B)$ is soft tripolar fuzzy $\Gamma$-subsemiring of $M$. By Definition[2.14], $(f, A) \wedge(g, B)=(f \wedge g, C)$, where $C=A \times B$.

Suppose $(a, b) \in C, x, y \in M$ and $\alpha \in \Gamma$. Then

$$
\begin{aligned}
\mu_{f \wedge g(a, b)}(x \alpha y \beta z) & =\mu_{f(a) \cap g(b)}(x \alpha y \beta z) \\
& =\min \left\{\mu_{f(a)}(x \alpha y \beta z), \mu_{g(b)}(x \alpha y \beta z)\right\} \\
& \geq \min \left\{\mu_{f(a)}(y), \mu_{g(b)}(y)\right\} \\
& =\mu_{f(a) \cap g(b)}(y) \\
& =\mu_{f \wedge g(a, b)}(y) . \\
\lambda_{f \wedge g(a, b)}(x \alpha y \beta z) & =\lambda_{f(a) \cap g(b)}(x \alpha y \beta z) \\
& =\min \left\{\lambda_{f(a)}(x \alpha y \beta z), \lambda_{g(b)}(x \alpha y \beta z)\right\} \\
& \leq \min \left\{\lambda_{f(a)}(y), \lambda_{g(b)}(y)\right\} \\
& =\lambda_{f(a) \cap g(b)}(y)
\end{aligned}
$$




$$
\begin{aligned}
& =\lambda_{f \wedge g(a, b)}(y) . \\
\delta_{f \wedge g(a, b)}(x \alpha y \beta z) & =\delta_{f(a) \cap g(b)}(x \alpha y \beta z) \\
& =\min \left\{\delta_{f(a)}(x \alpha y \beta z), \delta_{g(b)}(x \alpha y \beta z)\right\} \\
& \leq \min \left\{\delta_{f(a)}(y), \delta_{g(b)}(y)\right\} \\
& =\delta_{f(a) \cap g(b)}(y) \\
& =\delta_{f \wedge g(a, b)}(y) .
\end{aligned}
$$

Hence $(f, A) \wedge(g, B)$ is a soft tripolar fuzzy interior ideal of $\Gamma$-semiring $M$.

The following theorem proofs are similar to Theorem [3.4].

Theorem 3.5. If $(f, A)$ and $(g, B)$ are two tripolar fuzzy soft interior ideals over a $\Gamma$-semiring $M$ then $(f, A) \cup(g, B)$ is a tripolar fuzzy interior ideals of $\Gamma$-semiring $M$.

Theorem 3.6. If $(f, A)$ and $(g, B)$ are two tripolar fuzzy soft interior ideals over $a \Gamma$-semiring $M$ then $(f, A) \cap(g, B)$ is a tripolar fuzzy interior ideals of $\Gamma$-semiring M.

Theorem 3.7. If $(f, A)$ and $(g, B)$ are two tripolar fuzzy soft ideals over $\Gamma$-semiring $M$ then $(f, A) \cup(g, B)$ is a tripolar fuzzy soft ideal over $M$.

Proof. Suppose $(f, A)$ and $(g, B)$ are two tripolar fuzzy soft ideals over $\Gamma$-semiring $M$. Then by Definition [2.13], we have $(f, A) \cup(g, B)=(h, C)$, where $C=A \cup B$; and

$$
h(c)=f \cup g(c)= \begin{cases}f(c) & \text { if } c \in A \backslash B ; \\ g(c) & \text { if } c \in B \backslash A ; \\ f(c) \cup g(c) & \text { if } c \in A \cap B, \text { forall } c \in A \cup B .\end{cases}
$$

case(i) : If $c \in A \backslash B$ then $f \cup g(c)=f(c)$. Thus we have

$$
\begin{aligned}
\mu_{f \cup g(c)}(x+y) & =\mu_{f(c)}(x+y) \\
& \geq \min \left\{\mu_{f(c)}(x), \mu_{f(c)}(y)\right\} \\
& =\min \left\{\mu_{f \cup g(c)}(x), \mu_{f \cup g(c)}(y)\right\} . \\
\lambda_{f \cup g(c)}(x+y) & =\lambda_{f(c)}(x+y) \\
& \leq \max \left\{\lambda_{f(c)}(x), \lambda_{f(c)}(y)\right\} \\
& =\max \left\{\lambda_{f \cup g(c)}(x), \lambda_{f \cup g(c)}(y)\right\} . \\
\delta_{f \cup g(c)}(x+y) & =\delta_{f(c)}(x+y) \\
& \leq \max \left\{\delta_{f(c)}(x), \delta_{f(c)}(y)\right\} \\
& =\max \left\{\delta_{f \cup g(c)}(x), \delta_{f \cup g(c)}(y)\right\} . \\
\mu_{f \cup g(c)}(x \alpha y) & =\mu_{f(c)}(x \alpha y)
\end{aligned}
$$




$$
\begin{aligned}
\geq & \max \left\{\mu_{f(c)}(x \alpha y), \mu_{f(c)}(x \alpha y)\right\} \\
& =\max \left\{\mu_{f \cup g(c)}(x), \mu_{f \cup g(c)}(y)\right\} . \\
\lambda_{f \cup g(c)}(x \alpha y) & =\lambda_{f(c)}(x \alpha y) \\
& \leq \min \left\{\lambda_{f(c)}(x), \lambda_{f(c)}(y)\right\} \\
& =\min \left\{\lambda_{f \cup g(c)}(x), \lambda_{f \cup g(c)}(y)\right\} . \\
\delta_{f \cup g(c)}(x \alpha y) & =\delta_{f(c)}(x \alpha y) \\
& \leq \min \left\{\delta_{f(c)}(x), \delta_{f(c)}(y)\right\} \\
& =\min \left\{\delta_{f \cup g(c)}(x), \delta_{f \cup g(c)}(y)\right\} .
\end{aligned}
$$

case(ii) : If $c \in B \backslash A$ then $f \cup g(c)=g(c)$.

Since $g(c)$ is a tripolar ideal of $\Gamma$-semiring $M, f \cup g(c)$ is a tripolar ideal of $\Gamma$-semiring $M$.

case(iii) : If $c \in A \cap B$ then $f \cup g(c)=f(c) \cup g(c)$.

$$
\begin{aligned}
\mu_{f \cup g(c)}(x+y) & =\mu_{f(c) \cup g(c)}(x+y) \\
& =\max \left\{\mu_{f(c)}(x+y), \mu_{g(c)}(x+y)\right\} \\
& \geq \max \left\{\min \left\{\mu_{f(c)}(x), \mu_{f(c)}(y)\right\}, \min \left\{\mu_{g(c)}(x), \mu_{g(c)}(y)\right\}\right\} \\
& =\min \left\{\max \left\{\mu_{f(c)}(x), \mu_{g(c)}(x)\right\}, \max \left\{\mu_{f(c)}(y), \mu_{g(c)}(y)\right\}\right\} \\
& =\min \left\{\mu_{f(c) \cup g(c)}(x), \mu_{f(c) \cup g(c)}(y)\right\} \\
& =\min \left\{\mu_{f \cup g(c)}(x), \mu_{f \cup g(c)}(y)\right\}
\end{aligned}
$$

Similarly we can prove

$$
\begin{aligned}
\lambda_{f \cup g(c)}(x+y) \leq & \max \left\{\lambda_{f \cup g(c)}(x), \lambda_{f \cup g(c)}(y)\right\} \\
\delta_{f \cup g(c)}(x+y) \leq & \max \left\{\delta_{f \cup g(c)}(x), \delta_{f \cup g(c)}(y)\right\} . \\
\mu_{f \cup g(c)}(x \alpha y) & =\mu_{f(c) \cup g(c)}(x \alpha y) \\
& =\max \left\{\mu_{f(c)}(x \alpha y), \mu_{g(c)}(x \alpha y)\right\} \\
\geq & \max \left\{\max \left\{\mu_{f(c)}(x), \mu_{f(c)}(y)\right\}, \max \left\{\mu_{g(c)}(x), \mu_{g(c)}(y)\right\}\right\} \\
= & \max \left\{\max \left\{\mu_{f(c)}(x), \mu_{g(c)}(x)\right\}, \max \left\{\mu_{f(c)}(y), \mu_{g(c)}(y)\right\}\right\} \\
= & \max \left\{\mu_{f(c) \cup g(c)}(x), \mu_{f(c) \cup g(c)}(y)\right\} \\
= & \max \left\{\mu_{f \cup g(c)}(x), \mu_{f \cup g(c)}(y)\right\} \\
& \operatorname{Similarly} \operatorname{we} \operatorname{can} \operatorname{prove} \\
\lambda_{f \cup g(c)}(x \alpha y) \leq & \min \left\{\lambda_{f \cup g(c)}(x), \lambda_{f \cup g(c)}(y)\right\} \\
\delta_{f \cup g(c)}(x \alpha y) \leq & \min \left\{\delta_{f \cup g(c)}(x), \delta_{f \cup g(c)}(y)\right\} .
\end{aligned}
$$

Therefore, $f \cup g(c)$ is a tripolar fuzzy ideal of $M$.

Hence $(f, A) \cup(g, B)$ is a tripolar fuzzy soft ideal over $M$.

Corollary 3.1. If $(f, A)$ and $(g, B)$ are two tripolar fuzzy soft ideals over $\Gamma$-semiring $M$ then $(f, A) \cap(g, B)$ is a tripolar fuzzy soft ideal over $M$. 
Definition 3.4. Let $(f, A)$ and $(g, B)$ be two tripolar fuzzy soft sets over a $\Gamma$-semiring $M$. The the product $(f, A)$ and $(g, B)$ is defined as $(f, A) \circ(g, B)=(f \circ g, C)$, where $C=A \cup B$. Then we have $(f, A) \circ(g, B)=(h, C)$, where $C=A \cup B$; and

$$
\begin{gathered}
\mu_{(f \circ g)(c)}(x)=\left\{\begin{array}{lr}
\mu_{f(c)}(x), & \text { if } c \in A \backslash B ; \\
\mu_{g(c)}(x), & \text { if } c \in B \backslash A ; \\
\sup _{x=y \alpha z}\left\{\min \left\{\mu_{f(c)}(y), \mu_{g(c)}(z)\right\}\right\}, & \text { if } c \in A \cap B .
\end{array}\right. \\
\lambda_{(f \circ g)(c)}(x)= \begin{cases}\lambda_{f(c)}(x), & \text { if } c \in A \backslash B ; \\
\lambda_{g(c)}(x), & \text { if } c \in B \backslash A \\
\inf _{x=y \alpha z}\left\{\max \left\{\lambda_{f(c)}(y), \lambda_{g(c)}(z)\right\}\right\}, & \text { if } c \in A \cap B .\end{cases} \\
\delta_{(f \circ g)(c)}(x)= \begin{cases}\delta_{f(c)}(x), & \text { if } c \in A \backslash B ; \\
\delta_{g(c)}(x), & \text { if } c \in B \backslash A \\
\inf _{x=y \alpha z}\left\{\max \left\{\delta_{f(c)}(y), \delta_{g(c)}(z)\right\}\right\}, & \text { if } c \in A \cap B .\end{cases}
\end{gathered}
$$

Theorem 3.8. If $(f, A)$ and $(g, B)$ are tripolar fuzzy soft interior ideals over $\Gamma$-semiring $M$ then $(f, A) \circ(g, B)$ is a tripolar fuzzy soft interior ideal over $\Gamma$-semiring $M$.

Proof. Obviously $(f, A) \circ(g, B)$ is tripolar fuzzy soft $\Gamma$-subsemiring over $M$. Let $x, y, z \in M, \alpha, \beta \in \Gamma$.

By Definition [3.4], $(f, A) \circ(g, B)=(f \circ g, C)$, where $C=A \cup B$ and $c \in C, x \in M$. case(i) : If $c \in A \backslash B$ then

$$
\begin{aligned}
& \mu_{f \circ g(c)}=\mu_{f(c)} \\
& \lambda_{f \circ g(c)}=\lambda_{f(c)} \\
& \delta_{f \circ g(c)}=\delta_{f(c)} .
\end{aligned}
$$

Since $(f, A)$ is a tripolar fuzzy soft interior ideal over $M, f \circ g(c)$ is a tripolar fuzzy soft interior ideal of $M$.

case(ii) : If $c \in B \backslash A$ then

$$
\begin{aligned}
\mu_{f \circ g(c)} & =\mu_{g(c)} \\
\lambda_{f \circ g(c)} & =\lambda_{g(c)} \\
\delta_{f \circ g(c)} & =\delta_{g(c)} .
\end{aligned}
$$

Since $(g, B)$ is a tripolar fuzzy soft interior ideal over $M, f \circ g(c)$ is a tripolar fuzzy soft interior ideal of $M$.

case(iii) : If $c \in A \cap B$ then

$$
\mu_{f \circ g(c)}(x)=\sup _{x=a \alpha b}\left\{\min \left\{\mu_{f(a)}(x), \mu_{g(b)}(x)\right\} .\right.
$$




$$
\begin{aligned}
\mu_{f \circ g(c)}(x \alpha y \beta z) & =\sup _{x=a \alpha b}\left\{\min \left\{\mu_{f(a)}(x \alpha y \beta z), \mu_{g(b)}(x \alpha y \beta z)\right\}\right. \\
& \geq \sup _{x=a \alpha b}\left\{\min \left\{\mu_{f(a)}(y), \mu_{g(b)}(y)\right\}\right. \\
& =\mu_{f \circ g(c)}(y) . \\
\lambda_{f \circ g(c)}(x \alpha y \beta z) & =\inf _{x=a \alpha b}\left\{\max \left\{\lambda_{f(a)}(x \alpha y \beta z), \lambda_{g(b)}(x \alpha y \beta z)\right\}\right. \\
& \leq \sup _{x=a \alpha b}\left\{\max \left\{\lambda_{f(a)}(y), \lambda_{g(b)}(y)\right\}\right. \\
& =\lambda_{f \circ g(c)}(y) . \\
\delta_{f \circ g(c)}(x \alpha y \beta z) & =\inf _{x=a \alpha b}\left\{\max \left\{\delta_{f(a)}(x \alpha y \beta z), \delta_{g(b)}(x \alpha y \beta z)\right\}\right. \\
& \leq \inf _{x=a \alpha b}\left\{\max \left\{\delta_{f(a)}(y), \delta_{g(b)}(y)\right\}\right. \\
& =\delta_{f \circ g(c)}(y) .
\end{aligned}
$$

Hence $f \circ g(c)$ is a tripolar fuzzy interior ideal of $M$.

Therefore $(f, A) \circ(g, B)$ is a tripolar fuzzy interior ideal over $M$.

Theorem 3.9. Let $E$ be a perimeter set and $\sum_{E}(M)$ be the set of all tripolar fuzzy soft interior ideals over $\Gamma$-semiring $M$. Then $\left(\sum_{E}(M), \cup, \cap\right)$ forms complete distributive lattices along with the relation $\subseteq$.

Proof. Suppose $(f, A)$ and $(g, B)$ be soft interior ideals over $M$ such that $A \subseteq$ $E, B \subseteq E$.

By Theorems [3.6, 3.7], $(f, A) \cap(g, B)$ and $(f, A) \cup(g, B)$ are tripolar fuzzy soft interior ideals over $M$.

Obviously $(f, A) \cap(g, B)$ is lub of $\{(f, A),(g, B)\}$ and $(f, A) \cup(g, B)$ is glb of $\{(f, A)(g, B)\}$.

Every sub collection of $\sum_{E}(M)$ has lub and glb.

Hence $\sum_{E}(M)$ is a complete lattice.

We can prove $(f, A) \cap((g, B) \cup(h, C))=((f, A) \cap(g, B)) \cup((f, A) \cap(h, C))$. Therefore $\left(\sum_{E}(M), \cup, \cap\right)$ forms a complete distributive lattice.

\section{Conclusion}

Murali Krishna Rao [19] introduced the notion of tripolar fuzzy set in $\Gamma$-semigroup to be able to deal with tripolar information, as a generalization of fuzzy set, bipolar fuzzy set and intuitionistic fuzzy set. In this paper, we have introduced the notion of tripolar fuzzy soft subsemiring, tripolar fuzzy soft ideal, tripolar fuzzy soft interior ideals over $\Gamma$-semiring and studied some of their algebraic basic properties and relations between them. The results of this paper can be extended to different algebraic structures and it can be applied to decision making problems. 
Acknowledgment. The authors thank the referee(s) for their valuable suggestions which led to the improvement of the paper.

\section{REFEREN CES}

1. U. Acar, F. Koyuncu and B. TAnay: Soft sets and soft rings. Computers and Math. with Appli. 59 (2010), 3458-3463.

2. H. Aktas and N. Cagman: Soft sets and soft groups. Infor. Sci. . 177 (2007), 2726-2735.

3. K. T. Attensov: Intuitionistic fuzzy sets. Fuzzy sets and sys. 20 (1) (1986) $87-96$.

4. A. Aygunoglu and H. Aygun: Introduction to fuzzy soft group. Comput. Math. Appli. 58 (2009), 1279-1286.

5. F. Feng, Y. B. Jun and X. ZhaO: Soft semirings. Comput. Math. Appli. 56 (2008), 2621-2628.

6. J. Ghosh, B. Dinda and T. K. Samanta: Fuzzy soft rings and Fuzzy soft ideals. Int.J. P. App.Sci.Tech. 2(2) (2011), 66-74.

7. Y. B. JUN, H. S. KIM and K. J. LEE: Bipolar fuzzy translation in BCK/BCIalgebra. J. of the Chungcheong Math. Soc. (22) (3) (2009), 399-408.

8. KYUNG Ho KIM and Y. B. Jun: Intuitionistic fuzzy interior ideals of semigroups. Int. J. of Math. and Math. Sci. 27 (5) (2001), 261-267.

9. K. J. LEE: Bipolar fuzzy subalgebras and bipolar fuzzy ideals of BCK/BCIalgebras. Bull. Malays. Math. Soc. (32) (2009), 361-373.

10. K. J. LEE: Bipolar valued fuzzy sets and their applications. Proc. Int. Conf. on Intelligent Technologies, Bangkok, Thailand (2000), 307-312.

11. K. M. LEE: Comparison of interval valued fuzzy sets, intuitionistic fuzzy sets and bipolar valued fuzzy sets. J. Fuzzy Logic intell. Sys. (14)(2) (2004), 125-129.

12. H. Lehmer: A ternary analogue of abelian groups. Amer. J. of Math. 59 (1932), 329-338.

13. W. G. Lister: Ternary rings. Tran. of Amer. Math. Soc. 154 (1971), 37-55.

14. P. K. Maji, R. Biswas and A. R. Roy: Fuzzy soft sets. The J. of Fuzzy Math. 9(3) (2001), 589-602.

15. D. Molodtsov: Soft set theory-First results. Comput. Math. Appl. 37 (1999), 19-31.

16. M. Murali Krishna RaO: $\Gamma$-semirings-I. Southeast Asian Bull. of Math. 19(1) (1995), 49-54.

17. M. Murali Krishna RaO: Г-semirings-II. Southeast Asian Bull. of Math. 21(3) (1997), 281-287.

18. M. Murali Krishna RaO: Fuzzy soft $\Gamma$ - semiring homomorphism. Anl. of Fuzzy Math. and Info. 12(4) ( 2016), 479-489.

19. M. Murali Krishna RaO: Tripolar fuzzy interior ideals of $\Gamma-$ semigroup. Anl. of Fuzzy Math. and Info. 15(2) (2018), 199-206.

20. M. Murali Krishna RaO and B. Venkateswarlu: Tripolar fuzzy interior ideals of $\Gamma$-semiring. Asia Pacific J. of Math. 5( 2) (2018), 192-207. 
21. M. Murali Krishna Rao and B. Venkateswarlu: Regular $\Gamma$-incline and field $\Gamma$-semiring. Novi Sad J. Math. 45(2) (2015), 155-171.

22. N. NoBusAwA: On a generalization of the ring theory. Osaka. J.Math. 1 (1964), $81-89$.

23. A. Rosenfeld: Fuzzy groups. J. Math. Anal. Appl. 35 (1971), 512-517.

24. M. K. Sen: On $\Gamma$-semigroup. Proc. of Inter. Con. of Alg. and its Appl., Decker Publicaiton, New York (1981), 301-308.

25. H. S. VANDIVER: Note on a simple type of algebra in which cancellatin law of addition does not hold. Bull. Amer. Math. Soc. 40 (1934), 914-920.

26. L. A. ZADEH: Fuzzy sets. Information and control. 8 (1965), 338-353.

27. W. R. Zhang: Bipolar fuzzy sets. Proc. of Fuzzy-IEEE. (1998), 835-840.

M. Murali Krishna Rao

GITAM Institute of Sciences

Department of Mathematics

GITAM University

Visakhapatnam, A.P., India

mmarapureddy@gmail.com

B. Venkateswarlu (Corresponding Author)

School of sciences

Department of Mathematics

GITAM University

Bengaluru Rural- 562 163, Karnatka, India

bvlmaths@gmail.com 